\title{
Prognostic Impact of Inflammation-related Biomarkers on Overall Survival of Patients with Inoperable Malignant Pleural Mesothelioma
}

\author{
TAKEHIRO OTOSHI, YUKI KATAOKA, SAWAKO KAKU, REIKA IKI and MASATAKA HIRABAYASHI
}

\author{
Department of Respiratory Medicine, Hyogo Prefectural Amagasaki General Medical Center, Amagasaki, Japan
}

\begin{abstract}
Aim: The aim of the present study was to assess the prognostic utility of the pretreatment blood neutrophilto-lymphocyte ratio (NLR) and the C-reactive protein-toalbumin ratio (CAR) in patients with inoperable malignant pleural mesothelioma (MPM). Materials and Methods: The medical records of consecutive patients with histologically confirmed MPM from our hospital between January 2007 and August 2017 were retrospectively reviewed. The primary outcome was overall survival (OS). Univariate and multivariate analyses for the prognostic factors were performed using a Cox proportional hazards model. Results: A total of 143 patients with inoperable MPM were included. On multivariate analysis, pretreatment CAR was an independent factor associated with worse OS (hazard ratio $(H R)=1.72 ; 95 \%$ confidence interval $(C I)=1.11-2.67$; $p=0.016)$. However, NLR was not associated with OS in any of the analyses. Conclusion: CAR appears to be a prognostic factor in patients with inoperable MPM.
\end{abstract}

Malignant pleural mesothelioma (MPM) is a highly aggressive tumor that is associated with asbestos exposure (1). Most patients present with unresectable disease due to their advanced age or underlying comorbidities (2). Although platinum-based palliative chemotherapy in combination with pemetrexed has been approved as a first-line therapy for patients with inoperable MPM, the median overall survival (OS) of patients remains very poor (9 months) (3). A short-

This article is freely accessible online.

Correspondence to: Takehiro Otoshi, MD, Department of Respiratory Medicine, Hyogo Prefectural Amagasaki General Medical Center, 2-17-77 Higashinaniwa-cho, Amagasaki, Hyogo 660-8550, Japan. Tel: +81 664807000, Fax: +81 664807001, e-mail: takehiro.otoshi19850902@gmail.com

Key Words: C-Reactive protein-to-albumin ratio, malignant pleural mesothelioma, neutrophil-to-lymphocyte ratio, prognostic factor. life expectancy in conjunction with potential side-effects and only modest survival benefit from the currently available palliative chemotherapy leads to the importance of a caseby-case risk-benefit assessment before treatment is begun (4). Therefore, the identification of prognostic determinants is important when deciding the treatment plan for patients with inoperable MPM.

It is known that the systemic inflammatory response plays an important role in the development and progression of various cancer types, and it has been reported that one inflammation-related biomarker, the blood neutrophil-tolymphocyte ratio (NLR), is useful for predicting the prognosis of patients with cancer (5). It has also been suggested that chronic inflammation due to asbestos exposure plays a critical role in the development and progression of MPM, and previous studies reported that NLR was a good prognostic factor for predicting OS in patients with MPM (4, 6-8).

On the other hand, another inflammation-related biomarker, the blood C-reactive protein (CRP)-to-albumin ratio (CAR), has also been reported to be a good prognostic factor in various cancer types (9-15). In some of these studies, CAR and NLR were assessed simultaneously, and both of them were reported to be prognostic factors for OS in patients with operable non-small cell lung cancer and those with unresectable metastatic colorectal cancer $(9,11)$. However, in other types of cancer (metastatic nasopharyngeal carcinoma, surgical renal cell carcinoma, surgical gastric cancer), CAR was found to be an independent prognostic factor for OS, while NLR was not $(10,12,13)$. As for MPM, as far as we are aware, only one study reported the prognostic utility of CAR (16), and no previous study has compared the prognostic utility of CAR and NLR.

In this study, the aim was to assess the prognostic utility of pretreatment NLR and CAR in patients with inoperable MPM. In addition, as a subgroup analysis, the prognostic utility of these two biomarkers was evaluated in patients with inoperable MPM who received palliative chemotherapy. 


\section{Materials and Methods}

Study design. A retrospective study was performed at our hospital (700-bed teaching hospital in Amagasaki City, Japan). This retrospective study was in accordance with the The Strengthening the Reporting of Observational Studies in Epidemiology statement (17) and was approved by the Institutional Review Board of Hyogo Prefectural Amagasaki Medical Center (approval number 29-122). All procedures performed in this study were in accordance with the ethical standards of the institutional and national research committees and with the 1964 Helsinki Declaration and its later amendments or comparable ethical standards. The Institutional Review Board of our hospital waived the need for informed consent from patients involved in this study because of its retrospective design.

Patients, data collection, and definitions. The medical records of consecutive patients with histologically-confirmed MPM between January 2007 and August 2017 in our hospital were reviewed. Patients with MPM who underwent surgical treatment were excluded from this study as were patients who had active inflammatory disease (such as rheumatoid arthritis) or infection at the time of MPM diagnosis. Patients who were treated by chemotherapy or radiotherapy before the histological diagnosis of MPM, patients with MPM confirmed by autopsy, patients who had entered a clinical trial, patients with insufficient follow-up periods (patients with a followup period of less than 1 month after the diagnosis of MPM or who had received treatment at other hospitals after the diagnosis of MPM) were also excluded. The included patients were divided into two groups, those who had received palliative chemotherapy and those who had received best supportive care.

Skilled pathologists had confirmed the histological diagnosis of MPM, using immunostaining such as for calretinin, cytokeratin $5 / 6$, carcinoembryonic antigen, thyroid transcription factor-1, and epithelial membrane antigen. Histological types of MPM were classified as epithelioid and non-epithelioid (7). The stages of MPM were classified according to the International Mesothelioma Interest Group (IMIG) staging system (18), with classes I and II in one group, and classes III and IV in another group (4).

Clinical variables (age, sex), laboratory data at the time of MPM diagnosis, first-line chemotherapy, and Eastern Cooperative Oncology Group performance status (PS) were obtained from the medical records. The NLR was calculated by dividing the serum neutrophil count by the serum lymphocyte count (7). The CAR was calculated by dividing the serum CRP level by the serum albumin level (19). Age and PS were each classified into two groups (age $<70$ or $\geq 70$ years; PS $\leq 1$ or $>1$ ), according to a previous report (20).

The primary outcome was OS, defined by the length of time from the date of diagnosis to death. Patients who had not died or were lost to follow-up were censored when they were last known to be alive before 1 October 2017.

Data presentation and statistical analysis. Analyses were carried out using the statistical software, Stata ${ }^{\circledR}$ ver. 13.0 (Stata Corp., College Station, TX, USA). Data are summarized using means (standard deviation) for continuous variables and numbers or percentages for categorical variables.

OS was estimated using the Kaplan-Meier method, and differences in survival rates were evaluated by the log-rank test. Univariate and multivariate analyses of prognostic factors were performed using the Cox proportional hazards model. The cut-off levels of NLR and CAR were defined as 5 and 0.58 , respectively, according to previous reports $(1,16)$.

Multiple imputation was used to handle missing data because it was assumed that missing values occurred at random $(21,22)$. Twenty datasets were imputed by normal regression, and estimates from these datasets were combined using Rubin's rule (23). The imputed set was used to investigate the prognostic utility of each factor, while the complete-case set was also used for preparing Kaplan-Meier survival curves. In all cases, $p$-values of less than 0.05 were considered significant.

\section{Results}

Patient characteristics. Between January 2007 and August 2017, 210 consecutive patients with histologically confirmed MPM were seen at our hospital (Figure 1). Twenty-four surgically treated patients were excluded, and 186 patients with inoperable MPM were potentially eligible. From these 186 patients, patients with active inflammatory disease or infection at the time of diagnosis, patients treated by chemotherapy or radiotherapy before the histological diagnosis of MPM, MPM patients confirmed by autopsy, patients who had entered clinical trials, and patients with insufficient follow-up were excluded. Thus, 143 patients were finally included in this study (Figure 1).

The median follow-up for all included patients was 345 days. Of the 143 patients, 102 (71.3\%) died during followup. The clinical characteristics of patients included in this study are summarized in Table I. Among the 86 patients who received palliative chemotherapy, the first-line chemotherapy regimens were cisplatin plus pemetrexed $(n=67,78 \%)$, carboplatin plus pemetrexed $(n=15,17 \%)$, pemetrexed monotherapy $(\mathrm{n}=3,4 \%)$, and others $(\mathrm{n}=1,1 \%)$.

Univariate and multivariate analyses of variables associated with OS. To assess the prognostic utility of NLR and CAR in patients with inoperable MPM, univariate and multivariate analyses were performed using the imputed set. According to previous studies, histological type, stage, PS, and age were regarded as important confounding factors associated with OS $(20,24)$, and they were considered in the analyses. Whether patients received platinum-based palliative chemotherapy in combination with pemetrexed was also considered in the analyses. On univariate analysis, histological type, stage, PS, and CAR were associated with OS (Table II). Multivariate analysis showed that nonepithelioid histological type and CAR of greater than 0.58 were independent prognostic factors associated with poorer OS (Table II). NLR was not associated with OS on either univariate or multivariate analysis.

Subgroup analysis of patients treated with palliative chemotherapy. To assess the prognostic utility of NLR and CAR in patients with inoperable MPM who received 


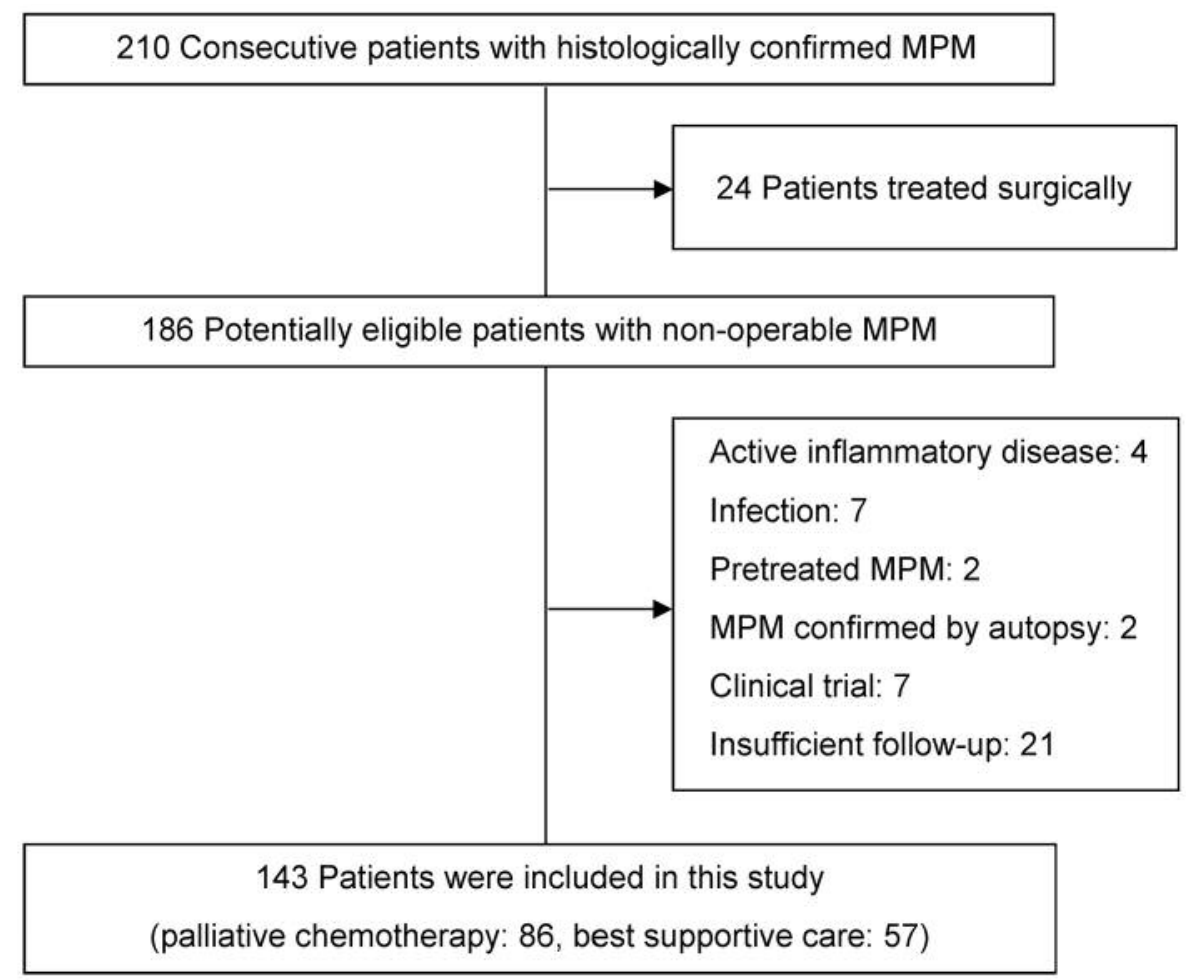

Figure 1. Flow chart of patient selection. MPM: Malignant mesothelioma.

Table I. The clinical characteristics of patients included in this study $(n=143)$.

\begin{tabular}{lccc}
\hline Variable & Palliative chemotherapy & Best supportive care & Total \\
\hline Patients, $\mathrm{n}$ & 86 & 57 & 143 \\
Mean age (SD), years & $70(8)$ & $76(7)$ & $72(8)$ \\
Gender (male), n (\%) & $65(76 \%)$ & $37(65 \%)$ & $102(71 \%)$ \\
Histological type (epithelioid), n (\%) & $43(50 \%)$ & $31(54 \%)$ & $74(52 \%)$ \\
Stage (I/II), n (\%) & $24(28 \%)(\mathrm{n}=1)^{\mathrm{a}}$ & $33(58 \%)(\mathrm{n}=7)^{\mathrm{a}}$ & $57(40 \%)(\mathrm{n}=8)^{\mathrm{a}}$ \\
PS (0/1), $\mathrm{n}(\%)$ & $77(90 \%)(\mathrm{n}=3)^{\mathrm{a}}$ & $46(81 \%)(\mathrm{n}=5)^{\mathrm{a}}$ & $123(86 \%)(\mathrm{n}=8)^{\mathrm{a}}$ \\
Platinum-based chemotherapy with pemetrexed, $\mathrm{n}(\%)$ & $82(95 \%)$ & $0(0 \%)$ & $82(57 \%)$ \\
Mean NLR (SD) & $4.0(7.4)(\mathrm{n}=4)^{\mathrm{a}}$ & $3.8(2.4)(\mathrm{n}=1)^{\mathrm{a}}$ & $3.9(6.0)(\mathrm{n}=5)^{\mathrm{a}}$ \\
Mean CAR (SD) & $1.06(1.46)(\mathrm{n}=6)^{\mathrm{a}}$ & $0.93(1.92)(\mathrm{n}=1)^{\mathrm{a}}$ & $1.01(1.65)(\mathrm{n}=7)^{\mathrm{a}}$ \\
\hline
\end{tabular}

SD: Standard deviation; CAR: blood C-reactive protein-to-albumin ratio; NLR: blood neutrophil-to-lymphocyte ratio; PS: Eastern Cooperative Oncology Group performance status. aNumbers in parentheses indicate the numbers of patients with imputed data.

palliative chemotherapy, subgroup analysis was performed using the imputed set. The median follow-up for patients treated with palliative chemotherapy was 351 days. Of the 86 patients treated with palliative chemotherapy, 68 (79.1\%) died during the follow-up period. According to a previous report, histological type, stage, PS, and age were considered in the multivariate analysis $(20,24)$. Whether patients received platinum-based palliative chemotherapy in combination with pemetrexed was also considered. On multivariate analysis, non-epithelioid histological type, $\geq 70$ years of age, absence of platinum-based chemotherapy with pemetrexed, and CAR of greater than 0.58 were prognostic factors for poorer OS, while NLR was not associated with OS (Table III).

Kaplan-Meier survival curves stratified by NLR and CAR. Kaplan-Meier survival curves stratified by NLR and CAR are shown in Figures 2 and 3, respectively. These survival curves indicated that high levels of CAR were associated 
Table II. Analysis of variables associated with overall survival.

\begin{tabular}{|c|c|c|c|c|}
\hline \multirow[t]{2}{*}{ Variable } & \multicolumn{2}{|c|}{ Univariate analysis } & \multicolumn{2}{|c|}{ Multivariate analysis } \\
\hline & Hazard ratio $(95 \% \mathrm{CI})$ & $p$-Value & Hazard ratio $(95 \% \mathrm{CI})$ & $p$-Value \\
\hline Histological type: Non-epithelioid $v s$. epithelioid & $2.23(1.50-3.31)$ & 0.0001 & $2.06(1.36-3.13)$ & 0.001 \\
\hline Stage: III/IV $v s . \mathrm{I} / \mathrm{II}$ & $1.55(1.02-2.36)$ & 0.039 & $1.59(0.99-2.55)$ & 0.054 \\
\hline PS: $2 / 3 / 4$ vs. $0 / 1$ & $1.85(1.06-3.21)$ & 0.030 & $1.69(0.96-2.95)$ & 0.068 \\
\hline Age: $\geq 70$ vs. $<70$ years & $1.05(0.71-1.57)$ & 0.81 & $1.41(0.88-2.25)$ & 0.15 \\
\hline Platinum-based chemotherapy with pemetrexed: No vs. yes & $1.02(0.68-1.53)$ & 0.91 & $1.26(0.78-2.04)$ & 0.35 \\
\hline NLR: $>5 v s . \leq 5$ & $1.22(0.74-2.01)$ & 0.43 & $1.06(0.62-1.80)$ & 0.83 \\
\hline CAR: $>0.58 v s . \leq 0.58$ & $1.94(1.30-2.91)$ & 0.001 & $1.72(1.11-2.67)$ & 0.016 \\
\hline
\end{tabular}

CAR: Blood C-reactive protein-to-albumin ratio; CI: confidence interval; NLR: blood neutrophil-to-lymphocyte ratio; PS: performance status.

Table III. Multivariate analysis of variables associated with overall survival in patients treated with palliative chemotherapy.

\begin{tabular}{lcc}
\hline Variable & Hazard ratio $(95 \%$ CI) & $p$-Value \\
\hline Histological type: Non-epithelioid $v s$. epithelioid & $1.93(1.15-3.26)$ & 0.013 \\
Stage: III/IV $v s$. I/II & $1.50(0.82-2.72)$ & 0.19 \\
PS: $2 / 3 / 4$ s. $0 / 1$ & $0.90(0.38-2.15)$ & 0.81 \\
Age: $\geq 70$ vs. $<70$ years & $1.75(1.00-3.05)$ & 0.049 \\
Platinum-based chemotherapy with pemetrexed: No $v s$. yes & $3.19(1.02-10.0)$ & 0.047 \\
NLR: $>5$ vs. $\leq 5$ & $1.07(0.54-2.11)$ & 0.84 \\
CAR: $>0.58$ vs. $\leq 0.58$ & $1.89(1.10-3.23)$ & 0.02 \\
\hline
\end{tabular}

CAR: Blood C-reactive protein-to-albumin ratio; CI: confidence interval; NLR: blood neutrophil-to-lymphocyte ratio; PS: performance status.

with poorer overall survival, while no difference in overall survival was seen between those with high and those with low levels of NLR.

\section{Discussion}

This study showed that CAR was a probable prognostic factor for OS in patients with inoperable MPM. Moreover, CAR was also a prognostic factor for OS in patients with MPM who had received palliative chemotherapy. On the other hand, NLR was not associated with OS in any of the analyses.

The present study has three important clinical differences from previous studies. Firstly, this is the first study to examine the prognostic utility of CAR and NLR simultaneously in patients with MPM. The results of the present study imply that CAR, but not NLR, is a probable inflammation-related biomarker for predicting the prognosis of patients with MPM. As for NLR, although some studies reported its prognostic utility $(4,6,7)$, the present study and another previous report did not show its prognostic utility in patients with MPM (1). It is thought that the differences in patient populations, treatment options, or cut-off values of NLR could have influenced the reported prognostic utility of NLR in each study. For example, in the studies that showed that NLR had significant prognostic utility, i) treatment data were not available for up to $17 \%$ of the patients (4); ii) patients previously treated with chemotherapy or patients who received non-standardized therapy (imatinib or thalidomide) were included (6); and iii) the cut-off value of NLR was defined as 3 based on the data specific to its patient population (7). To prevent bias from these factors, the present study excluded previously treated patients and patients who had entered a clinical trial, and a prespecified NLR cut-off value of 5 was used, as reported previously $(1,4)$. Secondly, this is the first study that assessed the prognostic utility of CAR in patients with inoperable MPM. In the previous unpublished study that assessed the prognostic utility of CAR in 83 patients with MPM, 27 patients (33\%) underwent surgery, and 56 (67\%) received only chemotherapy, while no patients who received only best supportive care were included; this study concluded that CAR was an independent predictor for OS (16). The present study did not include patients who underwent surgery because most of the patients presented with unresectable disease (Figure 1). Previous studies also reported that most patients with MPM presented with unresectable disease due to their advanced age or underlying comorbidities (2). In the present study, 57 (40\%) out of 143 patients with inoperable MPM received only best supportive 


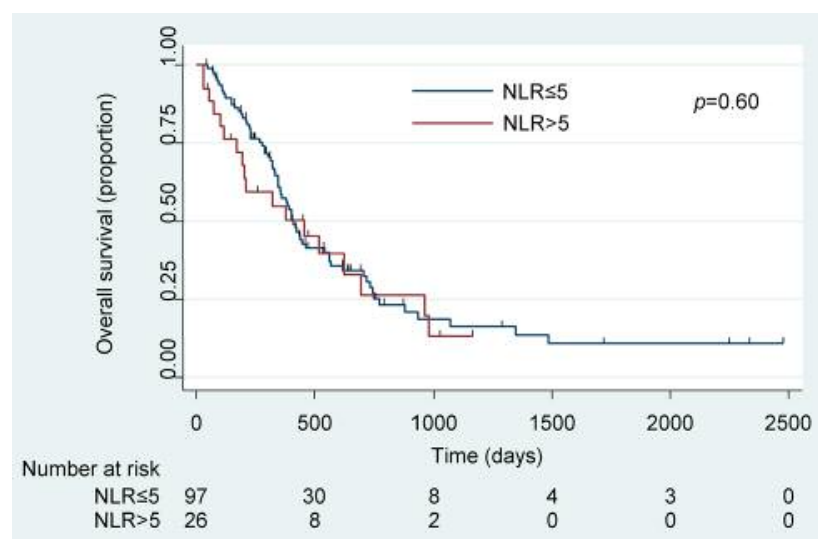

Figure 2. Kaplan-Meier survival curves for patients with malignant pleural mesothelioma stratified by blood neutrophil-to-lymphocyte ratio (NLR).

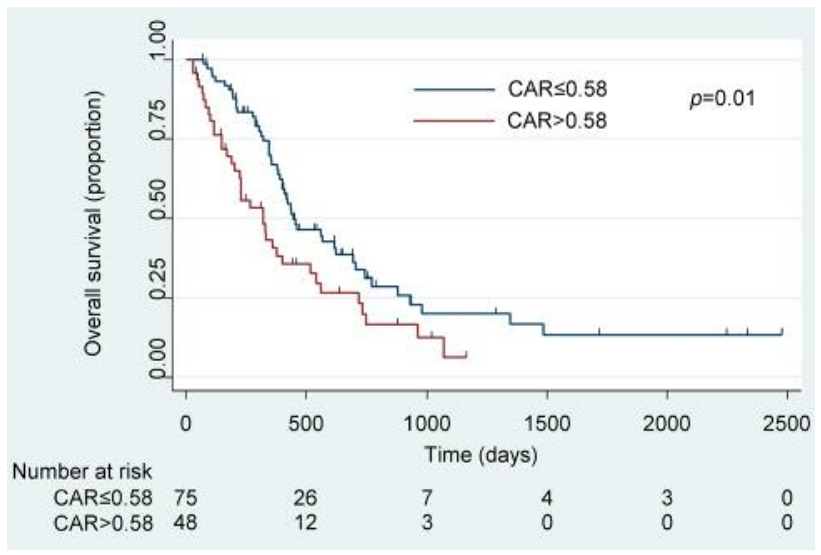

Figure 3. Kaplan-Meier survival curves for patients with malignant pleural mesothelioma stratified by blood C-reactive protein-to-albumin ratio $(C A R)$. care; the large number of such patients in the present study is comparable with another report that included 103 (38\%) out of 274 consecutive patients with MPM who received best supportive care (1). In this respect, the present study reflected the influence of the patients who were able to receive only best supportive care on the prognostic utility of inflammationrelated biomarkers. Thirdly, instead of selecting a cut-off level of CAR that was specific to the present patient population, this study used the cut-off value established previously (16). This means that in the present study it was possible to prevent overoptimistic estimates of test performance and externally validate the prognostic utility of CAR.

In this study, neither stage nor PS were a significant prognostic factor of OS, while a recent report regarding the prognostic factors in 191 patients with unresectable MPM showed that PS, but not stage, was an independent prognostic factor (25). Another previous study that included 274 patients with MPM also showed that PS, but not stage, was an independent prognostic factor (1). In the present study and these two previous studies, the hazard ratios for PS were approximately similar (1.69, 1.83 and 1.78 , respectively), and it is possible that the relatively small number of patients included in the present study could explain why PS was not found to be a significant prognostic factor.

The potential limitations of the present study are as follows. Firstly, NLR and CAR values were missing for some of the patients in this study because of its retrospective design, and multiple imputation was used to handle missing data. However, multiple imputation in this study was justified because it has been reported that this method reduces bias from missing data and improves the precision of estimates $(21,22)$. Secondly, there were no pre-specified criteria as to the treatment allocation for each patient included in this retrospective study. However, the treatment allocation in this study was reasonable because patients of younger age tended to receive palliative chemotherapy (Table I). Although this limitation reflects the real-world clinical setting, future multicenter, prospective studies with pre-specified treatment criteria are needed.

In summary, the results of the present study suggest that CAR is a probable prognostic marker when deciding the treatment plan in patients with inoperable MPM. Moreover, low levels of CAR might predict a better prognosis in patients with MPM who are able to receive palliative chemotherapy.

\section{Conclusion}

CAR is a probable prognostic factor in patients with inoperable MPM, and this needs to be confirmed in future large-scale, prospective studies.

\section{Conflicts of Interest}

The Authors declare that they have no conflict of interest in regard to this study. This research did not receive any specific grant from funding agencies in the public, commercial, or not-for-profit sectors.

\section{Acknowledgements}

The Authors would like to thank A. Sakurai, T. Imakita, S. Ikegaki, H. Matsumoto, E. Saito, T. Takemura, M. Shinomiya, M. Shimada, J. Nikaido, K. Hirano, T. Hirai, and K. Endo for caring for patients in this study.

\section{References}

1 Meniawy TM, Creaney J, Lake RA and Nowak AK: Existing models, but not neutrophil-to-lymphocyte ratio, are prognostic in malignant mesothelioma. Br J Cancer 109: 1813-1820, 2013.

2 Cihan YB, Ozturk A and Mutlu H: Relationship between prognosis and neutrophil: lymphocyte and platelet:lymphocyte ratios in patients with malignant pleural mesotheliomas. Asian Pac J Cancer Prev 15: 2061-2067, 2014. 
3 Beckett P, Edwards J, Fennell D, Hubbard R, Woolhouse I and Peake MD: Demographics, management and survival of patients with malignant pleural mesothelioma in the National Lung Cancer Audit in England and Wales. Lung Cancer 88: 344-348, 2015.

4 Pinato DJ, Mauri FA, Ramakrishnan R, Wahab L, Lloyd T and Sharma R: Inflammation-based prognostic indices in malignant pleural mesothelioma. J Thorac Oncol 7: 587-594, 2012.

5 McMillan DC: Systemic inflammation, nutritional status and survival in patients with cancer. Curr Opin Clin Nutr Metab Care 12: 223-226, 2009.

6 Kao SC, Pavlakis N, Harvie R, Vardy JL, Boyer MJ, van Zandwijk $\mathrm{N}$ and Clarke SJ: High blood neutrophil-tolymphocyte ratio is an indicator of poor prognosis in malignant mesothelioma patients undergoing systemic therapy. Clin Cancer Res 16: 5805-5813, 2010.

7 Kao SC, Vardy J, Chatfield M, Corte P, Pavlakis N, Clarke C, van Zandwijk $\mathrm{N}$ and Clarke S: Validation of prognostic factors in malignant pleural mesothelioma: a retrospective analysis of data from patients seeking compensation from the New South Wales Dust Diseases Board. Clin Lung Cancer 14: 70-77, 2013.

8 Chen N, Liu S, Huang L, Li W, Yang W, Cong T, Ding L and Qiu M: Prognostic significance of neutrophil-to-lymphocyte ratio in patients with malignant pleural mesothelioma: a metaanalysis. Oncotarget 8: 57460-57469, 2017.

9 Zhang F, Ying L, Jin J, Chen K, Zhang N, Wu J, Zhang Y and Su D: The C-reactive protein/albumin ratio predicts long-term outcomes of patients with operable non-small cell lung cancer. Oncotarget 8: 8835-8842, 2017.

10 Sun P, Chen C, Xia Y, Bi X, Liu P, Zhang F, Yang H, An X, Jiang $\mathrm{W}$ and Wang $\mathrm{F}$ : The ratio of $\mathrm{C}$-reactive protein/albumin is a novel inflammatory predictor of overall survival in cisplatinbased treated patients with metastatic nasopharyngeal carcinoma. Dis Markers 2017: 6570808, 2017.

11 Shibutani M, Maeda K, Nagahara H, Iseki Y, Hirakawa K and Ohira M: The significance of the $\mathrm{C}$-reactive protein to albumin ratio as a marker for predicting survival and monitoring chemotherapeutic effectiveness in patients with unresectable metastatic colorectal cancer. Springerplus 5: 1798, 2016.

12 Guo S, He X, Chen Q, Yang G, Yao K, Dong P, Ye Y, Chen D, Zhang Z, Qin Z, Liu Z, Xue Y, Zhang M, Liu R, Zhou F and Han $\mathrm{H}$ : The $\mathrm{C}$-reactive protein/albumin ratio, a validated prognostic score, predicts outcome of surgical renal cell carcinoma patients. BMC Cancer 17: 171, 2017.

13 Liu X, Sun X, Liu J, Kong P, Chen S, Zhan Y and Xu D: Preoperative $\mathrm{C}$-reactive protein/albumin ratio predicts prognosis of patients after curative resection for gastric cancer. Transl Oncol 8: 339-345, 2015

14 Shibutani M, Maeda K, Nagahara H, Iseki Y, Ikeya T and Hirakawa K: Prognostic significance of the preoperative ratio of C-reactive protein to albumin in patients with colorectal cancer. Anticancer Res 36: 995-1001, 2016.

15 Ide S, Toiyama Y, Okugawa Y, Oki S, Yasuda H, Fujikawa H, Yoshiyama S, Hiro J, Kobayashi M, Ohi M, Araki T and Kusunoki $\mathrm{M}$ : Clinical significance of C-reactive protein-to-albumin ratio with rectal cancer patient undergoing chemoradiotherapy followed by surgery. Anticancer Res 37: 5797-5804, 2017.
16 Takamori S, Toyokawa G, Tagawa T, Kinoshita F, Kozuma Y, Matsubara T, Haratake N, Akamine T, Hirai F, Takenoyama M, Ichinose $\mathrm{Y}$ and Maehara $\mathrm{Y}$ : The $\mathrm{C}$-reactive protein/albumin ratio is a novel significant prognostic factor in patients with malignant pleural mesothelioma. J Thorac Oncol 12: S2158, 2017.

17 von Elm E, Altman DG, Egger M, Pocock SJ, Gotzsche PC and Vandenbroucke JP: The Strengthening the Reporting of Observational Studies in Epidemiology (STROBE) statement: guidelines for reporting observational studies. PLoS Med 4: e296, 2007.

18 Rusch VW: A proposed new international TNM staging system for malignant pleural mesothelioma. From the International Mesothelioma Interest Group. Chest 108: 1122-1128, 1995.

$19 \mathrm{Xu} \mathrm{XL}, \mathrm{Yu} \mathrm{HQ}, \mathrm{Hu}$ W, Song Q and Mao WM: A novel inflammation-based prognostic score, the C-reactive protein/ albumin ratio predicts the prognosis of patients with operable esophageal squamous cell carcinoma. PLoS ONE 10: e0138657, 2015.

20 Nojiri S, Gemba K, Aoe K, Kato K, Yamaguchi T, Sato T, Kubota $\mathrm{K}$ and Kishimoto T: Survival and prognostic factors in malignant pleural mesothelioma: a retrospective study of 314 patients in the west part of Japan. Jpn J Clin Oncol 41: 32-39, 2011.

21 Little RJ, D’ Agostino R, Cohen ML, Dickersin K, Emerson SS, Farrar JT, Frangakis C, Hogan JW, Molenberghs G, Murphy SA, Neaton JD, Rotnitzky A, Scharfstein D, Shih WJ, Siegel JP and Stern H: The prevention and treatment of missing data in clinical trials. N Engl J Med 367: 1355-1360, 2012.

22 Sterne JA, White IR, Carlin JB, Spratt M, Royston P, Kenward MG, Wood AM and Carpenter JR: Multiple imputation for missing data in epidemiological and clinical research: potential and pitfalls. BMJ 338: b2393, 2009.

23 Rubin DB and Nathaniel S: Multiple imputation for interval estimation from simple random samples with ignorable nonresponse. J Am Stat Assoc 81: 366-374, 1986.

24 Kataoka Y, Yamamoto Y, Otsuki T, Shinomiya M, Terada T, Fukuma S, Yamazaki S, Hirabayashi M, Nakano T and Fukuhara $\mathrm{S}$ : A new prognostic index for overall survival in malignant pleural mesothelioma: the rPHS (regimen, PS, histology or stage) index. Jpn J Clin Oncol 45: 562-568, 2015.

25 Bille A, Krug LM, Woo KM, Rusch VW and Zauderer MG: Contemporary analysis of prognostic factors in patients with unresectable malignant pleural mesothelioma. J Thorac Oncol 11: 249-255, 2016.
Received January 4, 2018

Revised January 29, 2018

Accepted January 30, 2018 